
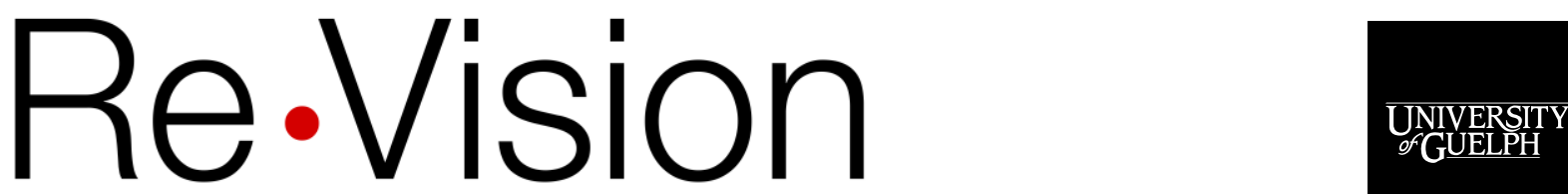

The Centre for Art and Social Justice

\title{
Fat reproductive justice: Navigating the boundaries of reproductive health care
}

\author{
Andrea LaMarre \\ Massey University \\ Carla Rice \\ University of Guelph \\ Katie Cook \\ Wilfrid Laurier University
}

May Friedman

Ryerson University

\begin{abstract}
"This is the peer reviewed version of the following article: LaMarre, A., Rice, C., Cook, K., \& Friedman, M. (2020). Fat reproductive justice: Navigating the boundaries of reproductive health care. Journal of Social Issues, $O(0), 1--25$, which has been published in final form at https://doi.org/10.1111/josi.12371. This article may be used for non-commercial purposes in accordance with Wiley Terms and Conditions for Use of Self-Archived Versions."
\end{abstract}

\section{Recommended citation:}

LaMarre, A., Rice, C., Cook, K., \& Friedman, M. (2020). Fat reproductive justice: Navigating the boundaries of reproductive health care. Journal of Social Issues, $0(0), 1--25$.

https://doi.org/10.1111/josi.12371 


\section{Fat Reproductive Justice: Navigating the Boundaries of Reproductive Health Care}

Andrea LaMarre, Carla Rice, Katie Cook \& May Friedman

\section{Acknowledgments}

We wish to thank all participants for their time, energy, and generosity in sharing their stories with us. This work was funded by a Canadian Institutes of Health Research (CIHR) Operating Grant \#137019.

\section{Abstract}

We explored the experiences of people in larger bodies seeking fertility and/or pregnancy care through a reproductive justice lens, integrating an understanding of weight stigma with an understanding of who has access to reproductive technologies, who is "allowed" to become pregnant, and the discourses that surround pregnancy. We conducted a thematic analysis of the narratives of 17 participants who had been labelled "overweight" or "obese" while pregnant and/or seeking reproductive healthcare related to fertility and/or pregnancy. Participants' narratives speak to experiences of being surveilled and controlled in medical settings; this surveillance and control negatively impacted their access to desired care. In order to receive the kinds of care they wanted, many participants had to become self-advocates. This self-advocacy speaks to resistance and "resilience"; we discuss how individualizing "resilience" represents an incomplete solution to navigating the shaming and blaming encounters participants experienced with healthcare providers. We argue for healthcare that is 
more caring and responsive to the needs of diverse individuals who are or who are seeking to become pregnant.

\section{Keywords}

reproductive health, reproductive justice, weight stigma, fatness, pregnancy

From advice on what one should eat, what one should wear, and how much one should exercise to unsolicited horror stories about the birth process, pregnancy is a fraught and fertile time for bodily surveillance and sanctioning. This is especially true in Westernized, neo-liberalized, and medicalized contexts where power operates in subtle and diffuse ways, including through expert recommendations about pregnancy and parenting that mobilize praise and censure to regulate behaviour. Parker and Pausé (2018) describe pregnancy as a time of "pre-emptive biopolitics" (p. 126), arguing that health discourses and regimes position fat ${ }^{1}$ pregnant people as posing significant risks to and burdens on society. Fat people who bear children are placed in the spotlight as being both socially and biologically to blame for any issue experienced by a fetus, an infant, or a child. As a result of discipline and control exerted in the name of population health, the experience of pregnancy may not always be the dreamy and expectant time it is popularly understood to be (Friedman, 2015). Rather than contributing to healthier pregnancies, shaming medical encounters harm those seeking care (Parker \& Pausé, 2019). In these contexts, fat people who are or seek to become pregnant often

\footnotetext{
${ }^{1}$ In this manuscript, we use the word "fat" when referring to the general category of bodies that are larger than idealized bodies in Western culture. Not all participants in our study used this word to describe themselves. We acknowledge the work of fat activists who have reclaimed the word "fat" as liberatory and to describe a physical and vital aspect of their embodied experiences of the world, in interaction and intersection with their other spaces of belonging (e.g., Cooper 2016; Wann, 1998).
} 
"experience discrimination, punishment, or mistreatment in maternity care" (Spence, 2013, p. 4).

In this paper, we use a reproductive justice framework coupled with an analysis of weight stigma to understand experiences of women and trans men who have been, or have attempted to become, pregnant. A reproductive justice frame helps us to explore how weight stigma, exerted in, on, and through participants' bodies and lives, limits "the complete physical, mental, spiritual, political, social, and economic well-being" (Ross \& Solinger, 2017, p. 70) of fat women and trans people in (and beyond) reproductive care settings. Reproductive justice work began with activism from women of colour, and especially Black women, who examined and spoke out about how systems of power work to exclude people from realizing a full range of human rights and freedoms, including rights to have or not have children, and the interrelated socioeconomic freedoms required to determine their preferred ways that this might be done (Ross, 2013; Zucker, 2014). As Solinger (n.d.) notes, reproductive choice-making becomes a privilege reserved for those with resources; only someone "with a sufficient bank account (and other personal resources such as a 'normal' genetic profile or a 'normal' IQ) has the makings of a legitimate mother" (p. 39).

Prior reproductive justice-oriented explorations of "choice," and the tensions inherent to assuming that choice is free, have tended to focus on the ways in which race, class, and sexuality delineate the margins of acceptability for parenting (e.g., Silliman, Gerber Fried, Ross \& Gutiérrez, 2016; Ross \& Solinger, 2017). Since its inception, the field of reproductive justice has grown to incorporate intersectional, multiissue articulations of the need to create systemic and discursive conditions such that 
people of all genders are able to realize reproductive freedom (Mason, 2013). To these important explorations, we add the layer of weight/size, engaging with narratives of those who have faced material and social constraints to reproductivity based on their size intersecting with other spaces of marginalization and belonging. In addition to analyzing participants' exclusions from realizing reproductive justice-or experiences of trauma, shame, and marginalization along the path to pregnancy-we examine how they negotiated and resisted systemic and ideological constraints, articulating these resistances in relation to broader efforts toward reproductive justice.

Within the Westernized world, fat bodies are sites of external and internal surveillance (Trainer, Brewis, \& Wutich, 2017). People who occupy bodies that fall outside limited zones of "normalcy" experience daily onslaughts of unsolicited comments about their corporeal characteristics and capacities (LaMarre \& Rice, 2016; Rice, 2007; 2014; Rinaldi, Rice, LaMarre, McPhail \& Harrison, 2017). Weight stigma is extremely prevalent and highly problematic, yielding self-blame, shame, and poor health outcomes (Puhl \& Brownell, 2001; Puhl \& King, 2013). Weight stigma carries negative emotions such as fear and distain that circulate in and across workplaces, healthcare, education, and society generally (Rinaldi, Rice, Kotow \& Lind, 2019). Despite the fact that "healthcare professionals are increasingly articulating the need for increased sensitivity in the treatment of obese patients" (Puhl \& Heuer, 2009, p. 948), negative attitudes and affects directed toward fat people continue to circulate, particularly when people are held personally responsible for their bodies being "obese" (Luck-Silkorski, Heller \& Phelan, 2017). This personal responsibility is often over-stated, despite a growing body of evidence that body size is not as firmly under an individual's control as 
might be assumed, particularly over time (Bacon \& Aphramor, 2011). Further, weight stigma is levelled strongly and persistently at people whose bodies are multiply othered: racialized, disabled, feminized, hetero and cis-normalized, and otherwise oppressed individuals have been subject to extreme and layered stigma when their bodies are also read as fat (Rice et al., forthcoming). Fat people navigating the realms of reproduction and fertility experience yet another layering of stigma, most notably in medicalized contexts (Parker, 2017). Many healthcare professionals hold fatphobic perspectives, including the misconception that fat people are personally responsible for reducing their weight to be "healthy" (e.g., Foster, Wadden, Makris, Davidson, Sanderson, Allison et al, 2003).

Stigma in healthcare settings is particularly pernicious as it can lead to poor mental and physical health outcomes for those targeted (Hatzenbuehler, Phelan, \& Link, 2013). Weight bias in the medical space can lead to avoidance of medical care (Ishmael \& Korf-Uzan, 2014), increased psychological burden, and lowered quality of care received (Thille, Friedman \& Setchell, 2017). Some researchers, whilst still framing "obesity" as a disease, have proposed "person-first" language (e.g., "person with obesity") to reduce stigma (e.g., Kyle \& Puhl, 2014; Wittert et al., 2015). However, others argue that this framing can perpetuate blame and stigma, and point to how studies exploring people's preferred terminologies are limited by who they include, for example, asking only those seeking "treatment" or exploring preferences amongst fat activists (Meadows \& Danielsdottir, 2016). At the very least, researchers argue that further studies into the effects of equating certain body sizes with disease are warranted. Even terms such as "overweight" raise questions about "over whose weight," 
(Wann, 1998, p. 19) with an unmarked norm lurking around the term's edges and impacting how people are perceived in medical/social arenas.

Fat pregnant bodies may be sanctioned subtly and explicitly by medical professionals, family members, friends, and strangers (McPhail, Bombak, Ward, \& Allison, 2016). Ongoing debates about fatness and fertility work to delineate and delimit weight thresholds for those attempting to access fertility treatment (Bomback et al., 2016). These debates circulate around a range of health concerns in fetuses and children, and many dictates around "fit motherhood" are directed at fat mothers and mothers-to-be (Bombak et al., 2016). Policies and debates about reproductive healthcare construct both material and social boundaries around who has access to reproductive services, technologies, care, and ultimately, reproduction itself. As an example, physicians across Canada have recently debated which body mass index (BMI) cut-offs to use when determining whether in vitro fertilization (IVF) should be offered to those classified as "obese" (Postmedia News, 2014). While IVF policies vary across the country, there is a resounding call to deny services to those inhabiting larger bodies on the grounds of fetal safety, maternal health, population health, and the possible (un)suitability of prospective parents (Friedman, 2015).

This rhetoric, articulated in policy debates and materialized in access, commingles with broader population-level concerns over who is a "fit" parent. Health authorities sound alarm bells about the health of future populations due to the assumed risks of fat bodies and recommend actions largely at the individual level to guard against these risks, such as focusing on possible child health risks associated with maternal body size (Bombak et al., 2016). These risks are "assumed" of, or attributed to, fat 
bodies in spite of emerging evidence that weight discrimination—rather than weight itself-has a strongly detrimental impact on health (Sutin, Stephan \& Terracciano, 2015). Weight stigma intersects with other social status markers used to restrict access to categories of parenthood and citizenship such as race, ethnicity, income and ability, often surfacing in reproductive care settings in the form of blame. This blame is leveraged in a way that is used to prop up control of bodies deemed "risky," as these people are assumed to be "unable" to make "correct" health choices (Parsons, Walsemann, Jones, Knopf \& Blake, 2016, p. 603). These "choices" are often framed as easily made and in simplistic terms (for instance, increasing exercise and decreasing caloric intake), with the assumption being that simply by letting people know that they must alter their behaviour they will be spurred into action (Ward \& McPhail, 2019).

Those who are fat and pregnant are deemed risky, despite mounting evidence that the risk related to body size has been misappropriated and can be better explained as an outcome of weight bias (DeJoy, Bittner \& Mandel, 2015). Risk discourses about reproductive "obesity" may stigmatize those who inhabit fat reproductive bodies by positioning them as unfit parents; this may prompt feelings of guilt, shame, and fear (Lupton, 2012). In communicating about risk to fat prospective parents, fetal and maternal health complications tend to be framed as inevitable and highly damagingand as inextricably tied to "obesity" (Ward \& McPhail, 2019). Further, risk discourses may intermingle and intensify when prospective parents occupy otherwise marked/marginalized embodiments due to class, race, disability, gender and more. Risk framing has significant impacts on well-being; interactions with stigmatizing healthcare providers may lead to emotional/psychological distress and physical complications, 
particularly when the only "solution" offered by providers is weight loss (Bernier \& Hanson, 2012) or when the practice guidelines that regulate them restrict fat prospective parents' access to care or make it conditional on weight loss, bariatric surgery, and other medicalized interventions.

In this paper, we explore the experiences of those who have been labelled "overweight" or "obese" in fertility and/or pregnancy care using a reproductive justice frame. Other work in the realm of weight stigma and reproduction, notably Parker (2014, 2018) and Parker \& Pausé $(2018,2019)$, Bombak et al. (2016), and McPhail et al. (2016) sets out convincing arguments for the presence of and need to counter weight stigma in fertility and pregnancy care. To this body of literature, we contribute work that takes up a reproductive justice lens, exploring affective and material constraints faced by those experiencing weight stigma in these settings. We asked: what kinds of care do women and trans people labelled "overweight" and "obese" encounter when seeking to become pregnant (or seeking care when already pregnant)? What is opened and what is constrained by systemic framings of fatness in fertility and pregnancy care? We were interested in the kinds of care that fat pregnant people were interested in receiving, how the care they received met or did not meet their expectations, and how they negotiated this care. In keeping with a reproductive justice lens, we focused on how fatness intersected with other spaces of social othering and belonging to open and/or constrain access to needed and desired healthcare before, during, and after pregnancy.

\section{Method}

Our study was initiated to explore weight stigma in reproductive care services in Canada across three provinces: Manitoba, Ontario, and Quebec; the 17 interviews we 
analyse here are from the Ontario site. The broad aim of the study was to understand the kind of reproductive healthcare that people classified as "obese" receive. The larger study also included interviews with healthcare providers, a policy analysis, and a videography project that engaged participants in creating short, first-person films. We received ethics approval through four university ethics boards, including the research ethics board at the institution from which the Ontario arm of our study was conducted, the University of Guelph.

\section{Reflexivity}

Throughout the research, we considered how our identities shaped our experiences and our analysis, in keeping with a reflexive approach to researchespecially research dealing with embodied experiences (Rice, 2009). We offer these reflections as a way to situate ourselves in the work we do. I (AL) am a White, cisgender, heterosexual, thin woman who passes as able-bodied; I have experiences of disordered eating and "recovery." Entering into this project, I became acutely aware of my childlessness and struggles with fertility, and how this impacted my relationship with participants' stories, as well as the affect that circulated within the interview room and in analysis. My thin privilege in particular intersected with participants' stories as we spoke; in some cases, my body entered into the room in a tacit way and at other times more explicitly, when participants would remark upon my size in relation to healthcare providers who had treated them badly.

I (CR) am a queer, White, fat woman (with an extended family of Indigenous kin) who has long identified as living with mindbody difference; through working in disability and fat activist communities I have come to see myself as situated ambiguously in and 
outside the categories of disability and fatness. I claim this in-between-ness in recognition that I do not face the same stigma and access barriers that those who are superfat or physically impaired confront. Though I have never given birth, I have a complex relationship with the category "mother": as a once working-class woman, I believed in my childbearing years that the only way I could "lift" myself out of poverty was to choose education over family; now in post-menopause and parenting three adult (step) children, I experience waves of sadness about this constrained "choice". Working on this research has brought these feelings of grief to the fore.

I, (MF), am a fat Brown woman sitting in complicated intersections around queerness and disability. I have birthed and now parent four children and experienced several pregnancy losses. Reproduction has been central to my identity. I think about fat and parenting, fat and reproduction nearly constantly; concern about presumptions about my health, my parenting, my capacity are coloured by the assumptions my body elicits in many public spaces. At the same time I delight in the ways my fat body provides comfort and care to my family.

I (KC) am a White, queer, genderqueer, fat person with a working-class background. I am not a parent and have never been pregnant; however, expectations around my fatness and reproduction are prevalent in my life, particularly in medical spaces. I engage in care work in various capacities, as a nanny, an auntie, and a postpartum doula-in-training. My ability to claim and find power in my fatness is inextricably connected to my gender, queerness, and chronic illness. I bring this positionality to my research and care work in the ways that I navigate interactions from a place of embodied relationality. 
Throughout the paper, we use the umbrella terms "people" and "parents" to signal that our study included both women and trans people. Recognizing that the language surrounding reproduction and parenting remains highly gendered and binary (e.g., mother and father), we use the term "mother" when referring to societal discourses such as mother-blame and to participants who self-identify as mothers. We refer to participants using the specific terms (e.g., man, woman, mother, father, parent) that they use to identify themselves. We recognize the varied terms that people mobilize to describe their weights and acknowledge the problematics of grouping people together using a term, such as fat or "obese," that may or may not resonate with their preferred ways of referring to their bodies. Given that the labels and measures used to assign weight and size are fraught, charged, contested, and fluid or dynamic, we have opted not to describe individual participants according to weight. However, since "obesity" denotes potential or actual pathology and "fatness" connotes a certain type of body/flesh, when writing about bodies as a collective we use "obesity" to refer to how fat bodies are defined by medical science, and "fat" where we describe people's embodied experiences.

\section{Participants}

We recruited participants using snowball sampling, including outreach to groups serving pregnant and parenting women and trans people, at community centres, via email list servs, and more. Despite strategic and targeted efforts at recruitment, we found it difficult to obtain a sample of participants with varied racial, socioeconomic, and other social locations/experiences. We also encountered issues with the language used in recruitment efforts; due to institutional ethics board requirements at one of our sites, 
all sites were required to use the word "obesity" in recruitment materials. We heard from several would-be participants that they found this framing to be alienating. For example, the use of medical language led some to wonder whether they fit the specific disease criteria or measures for "obesity." For others, the stigma carried by the negatively-charged, pathologizing language of "obesity" caused them to question whether they could safely participate in the study. Further, after receiving feedback about the restrictedness of the term "women" (as not all people who seek to become pregnant or become pregnant are women) we altered recruitment materials to more explicitly welcome trans people into the study, changing wording on study instruments from women to "women and trans people."

Of the 17 people we interviewed at the Ontario site, fifteen identified as women, and two as men; their average age at the time of the interview was 35 (range 28-44). The majority identified as White or did not specify an ethnoracial identity; two identified as White and Jewish, and one as Indigenous.

\section{Procedure}

Participants engaged in semi-structured interviews, which in general ranged from 20 to 60 minutes with one lengthier (2 hour) interview; the same interviewer (AL) conducted and recorded all interviews and later had them transcribed verbatim through a professional service. Participants were asked about their relationships with their bodies before, and during, and after (if relevant) pregnancy, about which healthcare providers they interacted with during fertility and/or pregnancy care and what these experiences were like, and about how they would envision ideal healthcare systems for fertility and reproductive health care. We undertook a thematic analysis (Braun \& 
Clarke, 2006) from a critical feminist perspective, focusing primarily on making meaning at the latent level—or exploring patterns in the data in relation to broader social discourses/dictates_including policies, practices, and people with which/whom participants interacted. In keeping with a reproductive justice lens, we attended to the extent to which participants could access/exercise "choices" to have or not have children, and to the control they had (or did not have) around birthing options (Ross \& Solinger, 2017; Zucker, 2014). We attended also to discourse-to the regulating effects of expert language-in attempting to control the weights and/or the reproductive decisions of pregnant people and parents-to-be, and to the counter-arguments mobilized by some participants in speaking back to medical views. Taking this analytic approach allowed us to identify common patterns in the data and to explore the meanings and structural force of themes in the context of participants' lives.

To conduct this analysis, Principal and Co-Investigators designed a descriptive code-list based on a first read of the data as a whole. This initial list identified relevant aspects of healthcare encounters, including interactions with specific types of providers and of reproductive services most salient to particular interviewees, and aspects of the experience, such as the feelings these generated. One team member $(\mathrm{KC})$ first coded the data, adding codes to the list as needed. Following initial coding, AL began to develop themes, exploring the patterns that we observed in the data in relation to the research objectives. She shared these with the writing team, who discussed/refined themes and the specific extracts provided. Rather than exact agreement on "truths" within the data, we sought to understand the data as a whole, the patterns within it, how 
this related to the theoretical perspectives and lenses we bring to the data based on our own social positionings (Braun \& Clarke, 2006; 2019).

Our study has a number of limitations, which we wish to name upfront rather than in its "traditional" position in the discussion section, as they are particularly salient within an article rooted in a reproductive justice frame. First among these is the limited diversity of the sample. This sample predominantly included White cis-gender women in their mid-thirties, with minor differences in socioeconomic status. This may have impacted the results in a number of ways, including the extent to which participants had access to services and their responses to experiences of stigma (e.g., ability to go elsewhere, to be connected, to self-advocate, etc.). This lack of diversity also has implications for research conducted using a reproductive justice frame, particularly in relation to the deep roots of reproductive justice work in the efforts of women of colour. Though we can explore the material and ideological conditions that excluded participants in our study from realizing reproductive freedom, we lack significant understanding of how intersecting spaces of marginalization might further entrench reproductive inequities in relation to size. In any research about bodies, we also acknowledge the challenge of using words to capture embodied experiences.

\section{Results}

Participants' narratives include internalization of confusing, conflicting, and stigmatizing discourses about weight; the need to "prove" efforts to "maintain a healthy body" despite inhabiting bodies whose health practices and weight patterns "surprised" healthcare providers; and the intersections of multiple marginalizations leading to harmful treatment in healthcare settings. Participants encountered a range of 
discrediting and injurious encounters. These included fear- and shame-inducing comments about weight; scepticism regarding participants' accounts of their eating and exercise; enforcement of weight loss prior to pregnancy or fertility treatment; and intense monitoring of body size and curtailment of weight gain while pregnant.

Experiences were not uniformly negative but were shaded with complexity in receiving (sometimes simultaneously) good and stigmatizing care and practicing or imagining resistance within a system that attempts to discipline fat, pregnant, and otherwise marked bodies through medical and social technologies and policies. Participants' accounts were inflected with suggestions for imbuing systems with compassion and thoughtfulness, rather than assumptions and stigma. Below, we explore three key thematic areas. The first of these is related to participants' experiences of bodily surveillance, control, and assumptions from healthcare providers (The Weight of Assumed Dysfunction). The second attends to problematic "readings" of "complex" bodies by healthcare providers that impacted material access to care (Compounding Limited Access to Care). Finally, we explore the need for self-advocacy in care that highlights the problematics of a homogenizing medical system that uses difference primarily as a way of demarcating "problems" and situating blame (Desiring Difference in Systems of Care).

\section{The Weight of Assumed Dysfunction}

Participants described weight stigmatizing encounters with healthcare providers that made them out to be individually responsible for their weight, which was associated with and/or deemed responsible for any issues they were encountering or could potentially encounter around pregnancy. Pathology was commonly inscribed onto 
participants' bodies. Advice around what to "do about" weight was often vague, and again relied on an individualist frame. Encounters with healthcare providers in reproductive healthcare settings frequently began with questions about or imperatives for weight loss, prior to any questions about health, wellness, and behaviour more generally. One illustration of this lies in how assumptions were made even about why someone might be seeking fertility care. Megan (all names are pseudonyms), a White, cis-gender queer woman, discussed her first appointment with a new healthcare provider upon attempting to get pregnant:

I had been with male partners before and I married a woman, so when we went to have this little one, we needed the help of a fertility clinic just to get the sperm and I walked in and they were like "oh your BMl's kind of high and [...] you probably have polycystic ovaries, and you're old," and like all of these things. And I'm look, I've been pregnant 100 times - it seems like - I constantly have to use double protection when I'm with men and like I don't have a fertility problem, and it was just like beating a dead horse until I finally had to find another practitioner who wasn't a jerk, yeah.

Megan sought assistance because she needed sperm in order to get pregnant, yet was greeted with assumptions about "why she couldn't get pregnant." However, Megan had been pregnant before, in relationships where sperm was a part of the equation. We might read heteronormativity into this encounter as it interacts with weight stigma; that is, Megan's provider assumed that were she not someone with a "high BMI," she would be able to get pregnant-which ignores the fact that she was in a relationship with a person who does not produce sperm. Other categories of "illness" 
are inscribed onto Megan's body, including PCOS, which was a common misdiagnosis given to participants in this study.

Assumptions made about participants' bodies in medical practice entrenched ambiguous feelings of responsibility or blame, even when there was no medical issue present. Pamela, a White, cis-gender woman, noted that after much testing, it was discovered that her partner had fertility issues and that her own body may not have been problematic, as doctors had assumed:

Results came in from [hospital] that we'd been waiting for. And I actually have fully functioning organs and my eggs are very happy. [...] horrible testing, blood work, medications, to make your body do stuff in huge amounts—I produce copious amounts of very healthy eggs. It may not be regular time periods, but I have very happy organs that have never had cysts or anything wrong with them.

Pamela describes the unnecessary testing and procedures that she endured because of a series of medical assumptions based on her body size and experiences with (in)fertility. Similarly, Scott, a White trans man, described,

The first midwife, the one I dumped, would say things like, 'Well, you know, you really should do the gestational diabetes test because larger people are much more likely...' and I'd be sort of like, 'well, yes, except that I don't have any of these other things', and I did test for gestational diabetes and was at the bottom end of the scale.

And Hanna, a White, cis-gender woman, recalled: 
[The obstetrician] assumed that I would develop high blood pressure, never did. He assumed that I would develop preeclampsia, didn't. I did get gestational diabetes, but it is what it is and that's a condition that can happen to anybody. And I ultimately did need a c-section but it was because of his position not because of my size.

Because of the assumptions made about the relationship between fertility, "healthy pregnancy" and body size, participants commonly described that they felt the need to "prove" their health status to providers via describing health behaviours and undergoing tests, and to explain or justify the reasons they experienced particular outcomes (e.g., a caesarean section).

Participants sometimes internalized the anti-fat messages they received, and fluctuated between seeing their bodies as good or problematic. This was compounded with the vague recommendations for weight loss that providers often issued, which typically left participants feeling as though their bodies were "the problem." For instance, Lindsey had an interaction with a fertility doctor who told her to "just lose weight," and said "I just kind of looked at her and shook my head... Because l've been trying to lose weight for many years, and if it was that easy, then it would've happened by now." Participants internalized these messages to greater or lesser degrees, some currently pursuing weight loss at the time of the interview, others having stopped dieting long before embarking on their pregnancy attempts.

Tied to the confusing and sometimes contradictory messages received about their bodies, and the imperative for them to "correct" their weights to have "successful pregnancies," participants' own accounts often contained contradictions related to how 
people were made individually responsible for their health, weight, and fertility. For instance, participants sometimes deemed weight loss attempts "successful" even as they offered reflections on problems with the way that links between health and weight were socially framed. Robyn, a White, cis-gender woman, said the following:

R: ...I did Herbal Magic ....and I had a lot of success with it. I lost probably close to 80 pounds in four months, but I didn't stick with it [...] the supplements that I was taking, they were probably laxatives and appetite suppressants, so it wasn't sustainable. As soon as you stop taking the supplements, the rest of it's not sustainable. [...] l'm thinking about doing it again, just to get the weight off, just for nothing else than, now l'm convinced that it does have something to do with my infertility. But [...] I don't understand how it impacts infertility. I think if I had a clear indication of why, like if obesity caused endometriosis and then endometriosis caused infertility, then I would see the connection. But I don't see the connection between infertility and obesity, especially since $90 \%$ of my friends are larger than I am and have two or three kids.

Robyn described the context around making the "choice" to follow such a dietary protocol, noting "I just wanted to lose weight" and "now I'm convinced that it does have something to do with my infertility." These reflections bring the medical view on her body into the picture; Robyn's extract in general points to confusing and contradictory accounts around whether her body is "a problem" or not, with doctors' perspectives impacting the extent to which she believes her body is the problem (note the "now" in her exploration of the link between infertility and weight). This linking then impacts the extent to which Robyn makes the "choice" to diet to improve her fertility. At the same 
time, she details problems with the state of scientific knowledge about this relationship; using examples of her larger friends who have multiple children, Robyn reflects that "I don't see the connection between infertility and obesity," speaking to the broader lack of consistent evidence about whether body size impacts fertility and pregnancy outcomes.

\section{Compounding Limited Access to Care}

Participants often experienced less-than-ideal fertility and/or pregnancy care and routinely encountered weight stigma that limited their access to the kinds of care they needed and desired. They articulated the constraints the system exerted on their ability to access care and how this interacted with their embodied experiences, as bodies were made out to be "troublesome" because of their difference. Healthcare providers' assumptions of pathology, based on a misread of their bodily differences, frequently disrupted participants' own desired trajectories for care. Commonly, the additional tests to which participants were subject led to decisions about care that did not conform with their wishes. Discussions around these alterations to plans of care were often not rooted in thorough explanations of how the system might accommodate or centre difference, but rather as matters of fact around a universalizing standard of care for bodies. Cheryl, a White, cis-gender woman, shared:

And because l'm obese, it's kind of like mandatory to meet [the anesthesiologist] because he has to determine if I'm eligible to get an epidural.

Interviewer (AL): Really?

Cheryl: Really. Interviewer (AL): What justification do they give you for that? 
Cheryl: That they might not be able to get the needle in, which I mean I was just over 200 pounds at the time. I weigh more than that now but it's just like maybe if I was, you know, four or five hundred pounds I could understand that maybe, maybe, that's a big maybe. But like really? I mean it's not like I have layers and layers of fat over my spine that you can't find the right spot to put the needle in. [laughs] So, he - after he did that he sat - we both sat down, he looked at me and said well, I'm going to tell you this because it's true. If you get an epidural you are going to have a $\mathrm{C}$ section. He goes I guarantee it because of your weight [...] And I, because I was so anti C section, I was like I felt like he had shot me, I was like - cause at the time I was really on the fence about epidurals. I really didn't want to get one. I didn't. I really - I'd read a lot about them. I was like no, no, no, no, no [...] And it also made me feel extremely fat shamed. It's like well you're fat you're going to get an epidural and a C section, I call it now, except he was right. I mean as judgmental as he was and all that, it was true. He could have said it in a way different way, but it was true. And yeah, it's like it's true because it's almost as if the minute you get that epidural you're on this downward spiral to the O.R. Like you just - it's automatic.

Cheryl's experience with the anesthesiologist was typical for many participants in the study who described the ways their wishes for care were not fulfilled due to the assumption of pathology related to size. She described feeling like the anesthesiologist framed her body as requiring intervention, likely beyond the epidural ("downward spiral to the OR"). This step of meeting with the anesthesiologist made Cheryl feel like she lost control over her birth plan ("you're going to get an epidural and a C section, I call it 
now"). In saying "except he was right" and discussing the fact that she did require medical attention, Cheryl reports feeling like this was a self-fulfilling prophecy and presented in a humiliating way.

For some participants, these assumptions related to size as it intersected with other ways their bodies were marked out as different, and worked together to complicate access to desired care. For instance, Rachel, an Indigenous, cis-gender woman, commented on the intersection between cultural competency/safety and weight stigma, describing how her care providers lacked understanding of her preferences and needs. She reflected on the challenge of bringing Indigenous practices into her care:

They don't see the person, they don't see the cultural layer on it and what their needs or wishes are that are respecting the culture. It's just not on their radar at all. So, that needs to be I think changed quite a bit.... I don't even know if that's just unique to Indigenous cultures or whether there's other cultures that kind of do other ceremonies like that as well. So, like I don't know but it would be nice if people were educated in being culturally competent in terms of providing care.

In Rachel's experience, non-Indigenous medical practitioners, including both doctors and midwives, were unaware of Indigenous cultural practices and decolonized approaches to healthcare delivery that would have made her feel safe and comfortable, including the ability to keep and plant her placenta after birth. Rachel also described the negative experience of being put on display during labour and delivery (saying "I felt really embarrassed having that many people in the room looking around at me"). Rachel's experiences of mainstream healthcare during pregnancy were generally very negative and embarrassing; this may have at least partially been mitigated by an 
increased awareness of Indigenous birthing knowledge and practices, and of what was required to deliver competent, decolonized practice. Here, as an Indigenous woman in a larger body, Rachel was made "hyper(in)visible," simultaneously put on display and dismissed (Gailey, 2014).

Bodies were often treated as multiply risky or problematic when they transcended what was expected in the medical encounter, which participants described in relation to not being able to access the kind of care they desired. Differences from the idealized fertile body collided with limited access to generate circumstances in which the bodyand its difference-was framed as the problem, rather than the system itself. Other participants recounted similar issues, sometimes adding limited geographic and financial access to the picture of being unable to access a kind of care that they desired. Nora, a White, cis-gender woman, shared that the time it took to see a doctor increased her anxiety about her age impacting her fertility, in addition to her weight:

It was the latency in terms of referral time to actually, you know, connecting with a doctor $[\ldots]$ I think that's the only clinic that served all of [province], and [other province] and parts of [third province]. So they draw from a huge area in terms of patients. And that was what part and parcel of the issue was. And no control over that. Like, I had no control over that. Like, I knew I was getting older but that didn't matter.

Having "no control" over her aging resonated with participants' experiences of being given little control over the process of seeking care in the first place; any bodily complexity was framed as something participants should be able to control, and yet, like 
aging, participants do not have as much control over the size of their bodiesparticularly in the long-term—as may be assumed (Bacon \& Aphramor, 2011).

It would be overly simplistic to assume that pregnancy itself is a women's issue only; importantly, not only women get pregnant and have babies. However, the spaces participants navigated at times belied their heteropatriarchal and colonizing roots. Participants' accounts reflected how even in spaces designed to be accessible and inclusive, vestiges of marginalization and a lack of understanding of gender nonnormativity persist, as Luke, a White trans man, noted:

The experiences were complicated. [Hospital] has done a good job at doing some things, like they have signage up all over the prenatal ward with images of trans people, particularly being like 'We serve trans people here. Watch your assumptions around who might be needing services.' And they've done a bunch of work institutionally to look at all of their stuff and do some work around queer and trans accessibility at that hospital. But also [this is my] job. I do health advocacy for trans people, so I also felt totally un-fazed by things.

As Luke reflects, even in spaces where staff make efforts to be gender-affirming, there is often a significant amount of self-advocacy required to facilitate positive experiences. Luke's work involves advocacy around trans issues; and yet, as he reflects, practitioners still made assumptions about gender, including by mis-gendering, and asking problematic questions. While Luke was used to fielding such questions and described himself as "unfazed," his account brings up the question of how to ensure that an awareness of gender diversity and fluidity extends more deeply into training, rather than simply being seen as add-on or procedural process. This raises the importance of 
making available care that is attentive to the complexity of bodies rather than inflected with assumptions about one size fitting all and concordant denial or complication of care when bodies do not fit expectations.

\section{Desiring Difference in Systems of Care}

Participants' narratives spoke to the importance of healthcare systems welcoming difference; for participants in this study, this kind of welcoming was often only reached through self-advocacy. Often, self-advocacy became a way that participants were able to access the kind of care they desired; after years of being misrepresented and misunderstood, many had reached a breaking point around problematic care that led them to be the ones to insist on centering care in the encounter. Given that self-advocacy often led to better treatment, questions around responsibility in the care apparatus remain: must fat pregnant people be strong selfadvocates in order to receive good care? As Amber, a White, cis-gender woman, noted:

I went into my first interactions with people in a defensive mode. I assumed that they would not accept me. So I would like there to be a more welcoming attitude that I shouldn't have to go in with my fists up kind of thing. I don't mind doing it and I'm good at it but, I worried about it every time. Every time I started an interaction l'd be like is this going to be the issue? Is it going to happen now?

Amber reflects on the need to act as a strong, self-protective person-to anticipate and prepare for a stigmatizing experience-in order to avoid or mitigate the effects of problematic encounters. We cannot speculate about what the likelihood of having such an experience was in her case, but this quote illustrates how participants who self-advocated also recognized that their lives might have been made significantly 
easier by the existence of safeguards (policies, training) against misrepresentation and mistreatment. Rachel commented on the "point of exhaustion" self-advocates often reach:

For me, the biggest part is to make sure that I always question people, always have a good enough answer why things are being done and continue to challenge why. That's what I would do differently the next time around. And to make sure that I was satisfied with the answer and was really clear about it and to not give up either. I think there's a point of exhaustion that happens and not to feel bad about it because there's only so much you can handle, right? I think having an advocate on the side would have been helpful too. So, my partner was there and did his job. But I think having a third party to be really strong and involved would have helped out a lot more too.

Reflecting that she might be a stronger self-advocate the second time around, Rachel also notes that "there's only so much you can handle." This is a compassionate response to those who may not be able to speak up as self-advocates in how it avoids a perspective that leads to the person being made responsible or blamed for all ill health that might result from a lifetime of encounters such as these.

Throughout the interviews, participants reflected on how they wished that systems would respond in more difference-attuned and intersectional ways, offering a space for them to be in their bodies surrounded by people who respect them, particularly during a uniquely vulnerable time in their lives. This might be achieved in a number of ways, including by providing healthcare professionals with better training, as Scott described: 
So medical training isn't ideal. I think it's very hard for people to figure out who can advocate for them if they don't feel able to advocate for themselves. That there isn't a system of patient advocacy... [H]aving midwives opted me out of a more medicalized system but I also know that lots of folks who want midwives can't get them and that there isn't enough midwifery here.

Participants were particularly interested in challenging the existing education related to inquiring about health status/practices in a non-stigmatizing way. One method might be through better education on interacting with fat patients/clients in affirming, respectful ways; for instance, providers need to learn how to ask about health behaviours without commenting on size, as Nora describes.

There needs to be more education done, specifically with doctors around how they talk to and interact with people who might be overweight or obese... and really, you're pointing out the obvious, you're telling me I'm fat, like, I don't know that. But I think there's ways of doing it in a way that, like ... "Are you healthy?" "Are you a healthy person?" "Do you smoke" ...they didn't ever ask me if I smoked or drank.

Participants also issued strong calls for welcoming the person in their wholeness and uniqueness in healthcare encounters: many reiterated the desire to be seen and cared for as a full person, rather than merely as a fat person. Kimberley, a White, cisgender woman, suggested that the treatment of fat people tends to produce a dichotomy between full human beings and fat people, positioning those labelled "obese" or "overweight" as less and other than fully human. 
What I would say is. . . people who are overweight know it, they don't need to be placated. It doesn't need to be a three-ring circus. You need to be caring, wanting them to feel better for themselves, not necessarily because it's the norm. You need to be helping them, it's not your job to make them like everybody else.

This recommendation was particularly potent in reproductive care, where participants' stories revealed multiple experiences of being treated as mysteries or even medical marvels. Their recommendations for system improvement hinged on the idea of developing a sense of common human-ness, while listening to and believing lived experience, and welcoming difference. By making space for uncertainty and difference, participants' accounts revealed that there needs to be more room for varied experiences before, during, and after fertility care and/or pregnancy. This may enable a deeper sense of caring, and better treatment, in healthcare around fertility and pregnancy.

\section{Discussion}

Participants' narratives, taken together, revealed ways in which body size and other intersecting spaces of exclusion/belonging impacted participants' access to difference-attuned and culturally-competent reproductive healthcare. As our analyses show, participants' bodies were automatically assumed to be problematic, and became sites of surveillance, control, and assumptions in medical settings. These readings of participants' bodies had material impacts on their access to the kinds of care they desired. Participants enacted a great deal of self-advocacy in order to receive better care. Faced with a system that they either expected would make accessing care challenging or that they found to be exclusionary through their experiences as described in the study, participants took on the labour of articulating their humanity in a 
dehumanizing system. These findings add to the growing body of literature on fat shaming and weight bias in fertility and prenatal care, pointing to the ways in which assumptions levelled in healthcare settings led to material restrictions from desired care and to participants' resistance and self-advocacy. This self-advocacy was either preemptive, borne of their prior experiences as fat patients in medical systems, or reflective, suggesting that future interactions with healthcare providers would be guided by their experiences. In taking a reproductive justice orientation to this work, we have interrogated the intersections between "risk," choice, and material access to care-and specifically, to the kind of care that those seeking to become pregnant or who are pregnant find affirming and conducive to wellbeing.

Despite often extremely negative experiences, many expressed gratitude for practitioners who offered empathy and support throughout their journeys-and even to those who were not particularly helpful. As social beings, people are taught to make "responsible" choices and ultimately hold themselves accountable for their social/health outcomes (Rose, 2007; Wachs \& Chase, 2013). Thus, a possible explanation for this gratitude is that those who have been oppressed can become indebted even to those who oppress them (Bourdieu, 2001) perhaps particularly when the desired outcome (in this case, having a child) is "achieved." Seeking out care might be framed as making a "responsible choice," and when it leads to the outcome desired, people may feel that any unpleasant effects that result from the encounter were necessary by-products. This appreciation of negative encounters can further entrench inequities by reinforcing power dynamics (Wachs \& Chase, 2013, p. 117). This reinforcement of a particular (stigmatized) social positioning may lead to a particular pregnancy experience that 
makes changing the system secondary to achieving an individualized result (i.e., having a baby). This shift might be "productive but not constructive" (Parker \& Pausé, 2019) in how it shames people into enacting modes of self-governance that, rather than leading to health, "perpetuate a cycle of struggle and negative self-regard" (p. 264). While our study demonstrates a similar struggle and ambiguous, sometimes contradictory experiences of body, power, and control, participants were not necessarily content to maintain the status quo, and they expressed the need for changes in healthcare systems with which they had interacted.

Significantly, while all participants confronted weight stigma, some reported marginalizing experiences that they attributed to size interacting with other differences, namely their gender, sexuality, geographic location, age, and Indigeneity. There is now ample literature to show that fat people in general experience stigma in healthcare systems (e.g., Lupton, 2012; Swift, Hanlon, El-Redy, Puhl, \& Glazebrook, 2013; Rinaldi, Rice, Kotow \& Lind, 2019) and that populations labelled as "at risk" for "obesity" (i.e., women, racialized peoples, those living in poverty) in particular confront stereotypes and scepticism about their health knowledge, behaviours, and embodiments (Ernsberger 2009; Fee 2006; Poudrier 2007; Rice, 2006). Our findings contribute to this robust body of literature and also highlight how weight stigma might gain special traction in reproductive care settings, where risk discourses about the weight and health of birthing individuals and offspring circulate widely, and where nothing less than the wellbeing, vitality, and strength of the next generation is thought to be at stake (Parker \& Pausé, 2018). It is here that injunctions against fat might find fertile ground, and that practices of "covert eugenics"-involving encouragement and enforcement of weight 
loss prior to pregnancy, discouragement and refusal of fertility treatment prior to weight loss, and curtailment of weight gain during pregnancy_trickle into healthcare interactions (Bombak et al., 2016). Due to the intensity and ubiquity of anti-fat rhetoric in all sectors of society, for our participants this sometimes occurred without providers' or participants' awareness or understanding.

It is interesting to consider this line of argumentation, taken up in other work around fat, pregnancy and parenting (e.g., Bombak et al., 2016; McPhail et al., 2016; Parker \& Pausé, 2019) particularly in relation to reproductive justice. One of the central flashpoints in the reproductive justice debates has been the problematics of certain kinds of abortion policies or practices which encourage or discourage abortion access based on class and race (Mason, 2013); for instance, Mason (2013) explores the debates around Margaret Sanger and Planned Parenthood, nuancing the varied perspectives on the relationship between racism, eugenics, and abortion in the United States and articulating the tensions that infuse reproductive justice work that must be grappled with to make space for complex, intersectional analyses. Indeed, many reproductive justice scholars before us have pointed to the ways in which reproductive choice and rights discourse is inflected with shades of eugenics (Price, 2010, p. 55), and these shades persist in reproductive healthcare inquiries that begin with weightbased screenings (Ward \& McPhail, 2019). Women of colour activist organizations, such as SisterSong, have added nuance to this debate by surfacing how "population control" measures have unduly targeted marginalized communities while simultaneously advocating access for what is required to achieve health. What such organizations advocate for includes abortion, birth control, and fertility/pregnancy services but also 
necessarily encompasses good healthcare generally and healthy living and working environments-as central tenets of the reproductive justice movement (Price, 2010). As Mason (2013) articulates, exploring the "untold histories of eugenics" is part and parcel of the work of the reproductive justice movement, "requiring an intersectional analysis of place as well as race, class, gender, citizenship, sexuality, physicality, and nationality" (p. 237) — to this we add, questions of size and age. Participants' stories unveil another layer of complexity in the fight for equity in access to reproductive healthcare across the spectrum, underscoring the need for this care to be not only available but free from judgment and undue influence in the direction of not having, or having, children.

\section{Practical Implications}

Participants expressed a desire for more accessible services, and for more variety and options in the services available to them in order to fully realize their reproductive rights and freedoms. Partly because of the negative experiences many had with certain providers, the limited options to which participants had access for possible future pregnancies became problematic. In all, participants were seeking healthcare providers who would listen to and hear them, before simply making assumptions about their bodies. This finding is consistent with recent research assessing the experiences of fat mothers, as well as care providers supporting fat pregnant patients (DeJoy, Bittner \& Mandel, 2016; Heslehurst et al., 2015). Further, participants' care was not always in line with their expectations; others have similarly commented on how fat pregnant people may feel pressure to pursue medical interventions or plans of care that did not comply with their wishes (Ward \& McPhail, 2019). Here, participants reflected on how their experiences of pregnancy and encounters with medical providers felt like a self- 
fulfilling prophecy or at the very least disempowering. Zielinski (2019) suggests that such negative interactions are not inevitable, calling for medical professionals to consider the ways in which they orient to fat patients in reproductive healthcare settings. Similarly, Parker (2017) orients to the vitalizing politics of compassionate and socially just care as a more firm ground on which to build healthy families and healthy societies. In keeping with these calls, our participants' experiences reveal the critical importance of deeply considering the entanglement of power and care (Lester, 2018).

Practically, participants recommended increased training for healthcare providers ranging from nurses to midwives to doctors to naturopaths, on weight, health, and other intersections. Healthcare providers might be trained, for instance, to not have weight enter the consultation room as a first point of entry into the conversation (Zielinski, 2019). Weight would need to be considered in relation to the implicit biases that health providers may retain in spite of stated weight neutrality; such biases persist even in the face of scientific knowledge to the contrary and thus may not easily be displaced (Ward \& McPhail, 2019). Fahs (2019) suggests that before training can be effective, we must contend with the deeply ingrained hatred of fatness that circulates in and beyond medical contexts (Rinaldi, Rice, Kotow \& Lind, 2019). Nuanced and thoughtful training would ideally result in more than a cursory awareness of the different ways in which bodies present in care settings. In keeping with a reproductive justice approach, training would be one step—alongside policy change, advocacy, changes to healthcare environments and increased access_-in building a movement toward a world in which everyone has the "human right to maintain personal bodily autonomy, have children, not 
have children, and parent the children we have in safe and sustainable communities" (SisterSong, n.d.).

It is a disheartening and perhaps unsurprising finding that many participants entered into the care setting expecting to be treated poorly, and thus often girded themselves to be strong self-advocates, which sometimes did result in better care. As some participants reflected, this should not be a requirement; ideally, healthcare spaces would not require this level of self-advocacy. However, in a neoliberal, biopedagogical, and one-size-fits-all setting, it is unsurprising that self-advocacy is required (McPhail et al., 2016). In order to move beyond this framework, there is a strong need for recognition of the contextuality and materiality of people's lives, and a deeper sense of common human-ness that looks beyond the "problems" of difference (or difference as a problem), reaching instead for openness to that which challenges taken-for-granted medical model assumptions and perhaps even creates something exciting and new.

Fahs (2019) imagines the possible resistances that might engender shifts in the medical landscape that so commonly disparages fat people. She proposes a radical approach to resistance, for instance, boycotts of fat shaming medical services, protests, and artistic and satirical interventions pointing to the inherent issues with discourses on "obesity," and more. For Fahs, promoting fat affirmative care means an explicit interrogation of the systemic roots of the oppression of fat people. This moves the responsibility away from the receiver of care to the provider, and would make the kind of self-advocacy work participants described less necessary. This kind of shift requires both tangible alterations to practice (e.g., changing communicated messages, altering physical environments, and considering the ways in which anti-fat discourse infuses 
messages everywhere from the community to the doctor's office; Ward \& McPhail, 2019) and radical changes to the social imaginary around fatness (Fahs, 2019).

Despite some limitations described in the methods section, most notably the lack of diversity, particularly in terms of race and ethnicity, this study makes a significant contribution to our understanding of the experiences of reproductive healthcare amongst those who are labelled "obese". Participants' stories unveiled deeplyengrained problematics inherent to healthcare systems. These results may help to inform approaches to exploring the relationships between weight, health, and pregnancy in a way that complicates relations, rather than inscribes linearity. Our findings also highlight the importance of complicating stigma-based narratives given the extent to which participants did—and continue to-internalize the blaming messages they received about their bodies. If, as a society, we wish to build systems that allow people to thrive and truly be well, we cannot continue to blame people for having bodies that often act, as bodies will, in unexpected ways. Participants' stories revealed the imperative of bringing care back into healthcare and fertile relationships into fertility care.

\section{References}

Bacon, L., \& Aphramor, L. (2011). Weight science: Evaluating the evidence for a paradigm shift. Nutrition Journal, 10, Article 9. https://doi.org/10.1186/1475-2891$\underline{10-9}$

Bernier, J. R., \& Hanson, Y. (2012). Overweight and obesity in pregnancy: A review of the evidence. Halifax, NS: Atlantic Centre of Excellence for Women's Health. Retrieved from http://www.pwhce.ca/pdf/overweightObesityPregnancy.pdf 
Braun, V., \& Clarke, V. (2006). Using thematic analysis in psychology. Qualitative Research in Psychology, 3(2), 77-101. https://doi.org/10.1191/1478088706qp063oa

Braun, V., \& Clarke, V. (2019). Reflecting on reflexive thematic analysis. Qualitative Research in Sport, Exercise, and Health, 11(4), 589-597. https://doi.org/10.1080/2159676X.2019.1628806

Bombak, A. E., McPhail, D., \& Ward, P. (2016). Reproducing stigma: Interpreting "overweight" and "obese" women's experiences of weight-based discrimination in reproductive healthcare. Social Science \& Medicine, 166, 94-101.

\section{https://doi.org/10.1016/i.socscimed.2016.08.015}

Bourdieu, P. (2001). Masculine domination. Cambridge: Polity Press.

Cook, K., LaMarre, A., Rice, C., \& Friedman, M. (2019). “This isn't a high-risk body”: Reframing risk and reducing weight stigma in midwifery practice. Canadian Journal of Midwifery Research and Practice.

Cooper, C. (2016). Fat activism. Bristol, UK: HammerOn Press.

DeJoy, S. B., \& Bittner, K. (2015). Obesity stigma as a determinant of poor birth outcome in women with high BMI: A conceptual framework. Maternal and Child Health Journal, 19(4), 693-699. https://doi.org/10.1007/s10995-014-1577-x

DeJoy, S. B., Bittner, K., \& Mandel, D. (2016). A qualitative study of the maternity care experiences of women with obesity: "More than just a number on the scale". Journal of Midwifery \& Women's Health, 61(2), 217-223. https://doi.org/10.1111/jmwh.12375

Ernsberger, P. (2009). Does social class explain the connection between weight and health? In E. Rothblum \& S. Solovay (Eds.), The fat studies reader (pp. 25-36). New York, NY: NYU Press.

Fahs, B. (2019). Fat and furious: Interrogating fat phobia and nurturing resistance in medical framings of fat bodies. Women's Reproductive Health, 6(4), 245-251, https://doi.org/10.1080/23293691.2019.1653577

Foster, G. D., Wadden, T. A., Makris, A. P., Davidson, D., Swain Sanderson, R., Allison, D. B., \& Kessler, A. (2003). Primary care physicians' attitudes about obesity and 
its treatment. Obesity Research, 11(10), 1168-1177.

https://doi.org/10.1038/oby.2003.161

Friedman, M. (2015). Reproducing fat-phobia: Reproductive technologies and fat women's right to mother. Journal of the Motherhood Initiative, 5(2), 27-41. Retrieved from https://jarm.journals.yorku.ca/index.php/jarm/article/view/39755

Gailey, J. A. (2014). The hyper(in)visible fat woman: Weight and gender discourse in contemporary society. London, UK: Palgrave Macmillan.

Hatzenbuehler, M. L., Phelan, J. C., \& Link, B. G. (2013). Stigma as a fundamental cause of population health inequalities. American Journal of Public Health, 103(5), 813-21. https://doi.org/10.2105/AJPH.2012.301069

Heslehurst, N., Dinsdale, S., Sedgewick, G., Simpson, H., Sen, S., Summerbell, S.D., \& Rankin, J. (2015). An evaluation of the implementation of maternal obesity pathways of care: A mixed methods study with data integration. PLOS ONE, 10(5), 1-34. https://doi.org/10.1371/journal.pone.0127122

Ishmael, K. \& Korf-Uzan, K. (2014). Addressing weight bias and stigma in health care. Visions: BC's Mental Health and Substance Use Journal, 9(4), 18-19. Retrieved from https://www.heretohelp.bc.ca/addressing-weight-bias-and-stigma-healthcare

Kyle, T. K., \& Puhl, R. M. (2014). Putting people first in obesity. Obesity (Silver Spring), 22(5), 1211. https://doi.org/10.1002/oby.20727

LaMarre, A., \& Rice, C. (2016). Normal eating is counter-cultural: Embodied experiences of eating disorder recovery. Journal of Community and Applied Social Psychology, 26(2), 136-149. https://doi.org/10.1002/casp.2240

Lester, R. J. (2018). Ground zero: Ontology, recognition, and the elusiveness of care in American eating disorders treatment. Transcultural Psychiatry, 55(4), 516-533. https://doi.org/10.1177/1363461516674874

Luck-Silkorski, C., Riedel-Heller, S. G., \& Phelan, J. C. (2017). Changing attitudes toward obesity - Results from a survey experiment. BMC Public Health, 17, 373. https://doi.org/10.1186/s12889-017-4275-y 
Lupton, D. (2012). 'Precious cargo': Foetal subjects, risk and reproductive citizenship. Critical Public Health, 22(3), 329-40. https://doi.org/https://doi.org/10.1080/09581596.2012.657612

Mason, C. (2013). How not to pimp out reproductive justice: Adventures in education, activism, and accountability. Frontiers: A Journal of Women Studies, 34(3), 226241. Retrieved from https://www.law.berkeley.edu/phpprograms/centers/crri/zotero/loadfile.php?entity key=6VH2BNWI

McPhail, D., Bombak, A., Ward, P., \& Allison, J. (2016). Wombs at risk, wombs as risk: Fat women's experiences of reproductive care. Fat Studies, 5(2), 98-115. https://doi.org/10.1080/21604851.2016.1143754

Meadows, A., \& Daníelsdóttir, S. (2016). What's in a word? On weight stigma and terminology. Frontiers in Psychology, 7, Article 1527. https://doi.org/10.3389/fpsyg.2016.01527

Parker, G., \& Pausé, C. (2018). Pregnant with possibility: Negotiating fat maternal subjectivity in the "war on obesity." Fat Studies, 7(2), 124-134. https://doi.org/10.1080/21604851.2017.1372990

Parker, G., \& Pausé, C. (2019). Productive but not constructive: The work of shame in the affective governance of fat Pregnancy. Feminism \& Psychology, 29(2), 250268. https://doi.org/10.1177/0959353519834053

Parker, G. (2017). Shamed into health? Fat pregnant women's views on obesity management strategies in maternity care. Women's Studies Journal, 31(1), 2233. Retrieved from http://www.wsanz.org.nz/journal/docs/WSJNZ311Parker2233.pdf

Parsons, A. A., Walsemann, K. M., Jones, S. J., Knopf, H., \& Blake, C. E. (2016). The influence of dominant obesity discourse on child health narratives: A qualitative study. Critical Public Health, 5, 602-614. https://doi.org/10.1080/09581596.2016.1164298

Postmedia News. (2014). Should there be a weight cutoff for IVF? Firestorm of debate over denying fertility treatments to obese women. National Post. Retrieved from https://nationalpost.com/health/should-there-be-a-weight-cutoff-for-ivf-firestormof-debate-over-denying-fertility-treatments-to-obese-women 
Poudrier, J. (2007). The geneticization of Aboriginal diabetes and obesity: Adding another scene to the story of the thrifty gene. Canadian Review of Sociology/Revue Canadienne de Sociologie 44, 237-261. https://doi.org/10.1111/j.1755-618X.2007.tb01136.x

Price, K. (2010). What is reproductive justice? How women of color activists are redefining the pro-choice paradigm. Meridians, 10(2), 42-65. https://doi.org/10.2979/meridians.2010.10.2.42

Puhl, R., \& Brownell, K. D. (2001). Bias, discrimination and obesity. Obesity Research, 9(12), 788-805. https://doi.org/10.1038/oby.2001.108

Puhl, R. M., \& Heuer, C. A. (2009). The stigma of obesity: A review and update. Obesity, 17(5), 941-964. https://doi.org/10.1038/oby.2008.636

Puhl, R. M., \& King, K. M. (2013). Weight discrimination and bullying. Best Practice \& Research Clinical Endocrinology \& Metabolism, 27(2), 117-127. https://doi.org/10.1016/.j.beem.2012.12.002

Rice, C., Pendleton Jiménez, K., Harrison, E., Robinson, M., Rinaldi, J., LaMarre, A., \& Andrew, J. (forthcoming). Bodies at the intersection: Reconfiguring intersectionality through queer women's complex embodiments. Signs: A Journal of Women in Culture and Society.

Rice, C., (2014). Becoming women: The embodied self in image culture. Toronto, CAN: UT Press.

Rice, C. (2009). Imagining the other? Ethical challenges of researching and writing women's embodied lives. Feminism \& Psychology, 19(2), 245-266.

Rice, C. (2007). Becoming the fat girl: Emergence of an unfit identity. Women's Studies International Forum, 30(2), 158-174. https://doi.org/10.1177/0959353509102222

Rice, C. (2006). Out from under the occupation: Transforming our relationships with our bodies. In Medavarski, A., \& Cranney, B. (Eds.), Canadian woman studies: An introductory reader ( $2^{\text {nd }}$ ed., pp. 411-423). Toronto, CAN: Inanna Press.

Rinaldi, J., Rice, C., Kotow, C., \& Lind, E. (2019). Mapping the circulation of fat hatred. Fat Studies, 1-14. https://doi.org/10.1080/21604851.2019.1592949 
Rinaldi, J., Rice, C., LaMarre, A., McPhail, D. \& Harrison, E. (2017). Fatness and failed citizenship. Somatechnics, 7(2), 218-233.

https://doi.org/10.3366/soma.2017.0219)

Rose, N. (2007). The politics of life itself: Biomedicine, power, and subjectivity in the twenty-first century. Princeton, NJ: Princeton University Press.

Ross, L. J. (2013). The color of choice: White supremacy and reproductive justice. Retrieved from https://www.racialequitytools.org/resourcefiles/The-Color-ofChoice----Public-Version-with-footnotes-1.pdf

Ross, L. (2006). Understanding reproductive justice: Transforming the pro-choice movement. Off Our Backs, 36(4), 14-19. Retrieved from https://d3n8a8pro7vhmx.cloudfront.net/rrfp/pages/33/attachments/original/14564 25809/Understanding RJ Sistersong.pdf?1456425809

Ross, L., \& Solinger, R. (2017). Reproductive justice: An introduction. Oakland, CA: University of California Press.

Salmon, A., (2011). Aboriginal mothering, FASD prevention and the contestations of neo-liberal citizenship. Critical Public Health 21(2), 165e178. https://doi.org/10.1080/09581596.2010.530643

Silliman, J., Gerber Fried, M., Ross, L., \& Gutiérrez, E. (2016). Undivided rights: Women of color organizing for reproductive justice. Chicago: Haymarket Books.

SisterSong. (nd). What is reproductive justice? SisterSong. Retrieved from https://www.sistersong.net/reproductive-justice/

Swift, J. A., Hanlon, S., El-Redy, L., Puhl, R. M., \& Glazebrook, C. (2013). Weight bias among UK trainee dietitians, doctors, nurses and nutritionists. Journal of Human Nutrition and Dietetics, 26(4), 395-402. https://doi.org/10.1111/jhn.12019

Solinger, R. (n.d.). The incompatibility of neoliberal "choice" and reproductive justice. In Reproductive justice briefing book: A primer on reproductive justice and social change (pp. 39-40). Retrieved from https://www.law.berkeley.edu/phpprograms/courses/fileDL.php?fID=4051

Spence, R. (2013). Reproductive justice and childbearing women. National women's health network: The women's health activist. Retrieved from https://nwhn.org/reproductive-justice-and-childbearing-women/ 
Sutin, A.R., Stephan, Y., \& Terracciano, A. (2016). Weight discrimination and risk of mortality. Psychological Science, 26(11), 1803-1811. https://doi.10.1177/0956797615601103

Thille, P., Friedman, M., \& Setchell, J. (2017). Weight-related stigma and health policy. CMAJ, 189, E223-4. https://doi.org/10.1503/cmaj.160975

Trainer, S., Brewis, A., \& Wutich, A. (2017). Eating in the panopticon: Surveillance of food and weight before and after bariatric surgery. Medical Anthropology, 36(5), 500-514. https://doi.org/10.1080/01459740.2017.1298595

Wachs, F. L., \& Chase, L. F. (2013). Explaining the failure of an obesity intervention: Combining Bourdieu's symbolic violence and the Foucault's microphysics of power to reconsider state interventions. Sociology of Sport Journal, 30, 111-131. https://doi.org/10.1123/ssj.30.2.111

Wann, M. (1998). Fat! So? Because you don't have to apologize for your size. Berkeley, CA: Ten Speed Press.

Ward, P., \& McPhail, D. Fat shame and blame in reproductive care: Implications for ethical health care interactions. Women's Reproductive Health, 6(4), 225-241. https://doi.org/10.1080/23293691.2019.1653581

Wittert, G. A., Huang, K-C., Heilbronn, L. K., Kyle, T. K., \& Puhl, R. M. (2015). Supporting the call out for people first language in obesity. Obesity Research and Clinical Practice, 9(4), 309. https://doi.org/10.1016/j.orcp.2015.08.008

Zielinski, R.E. (2019). BMI and pregnancy/childbirth: Risk reduction or fat shaming? Women's Reproductive Health, 6(4), 242-244. doi:10.1080/23293691.2019.1653580

Zucker, A.N. (2014). Reproductive justice: More than choice. Analyses of Social Issues and Public Policy, 14(1), 210-213. doi:10.1111/asap.12059 